\title{
Late-onset Mitochondrial Cardiomyopathy Triggered by Anticancer Treatment
}

\author{
Takashi Yamasaki, Kenji Yanishi, Shuhei Tateishi, Naohiko Nakanishi, Kan Zen, \\ Takeshi Nakamura, Tetsuhiro Yamano, Hirokazu Shiraishi, \\ Takeshi Shirayama and Satoaki Matoba
}

\begin{abstract}
We report the case of a 62-year-old woman with a history of bilateral hearing impairment, who developed mitochondrial cardiomyopathy after chemotherapy. The patient underwent postoperative cisplatin chemotherapy after the surgical treatment of cervical cancer. The systolic function of her left ventricle decreased significantly. A tissue examination of the left ventricle revealed mitochondrial cardiomyopathy. Genetic testing revealed mutations in mitochondrial 3,243 A $\rightarrow$ G. Nine hundred fifty-five individual mutations were identified by next-generation sequencing. Since cardiovascular complications are the second leading cause of morbidity and mortality in patients undergoing cancer treatment, mitochondrial cardiomyopathy should be considered a potential cause of heart failure.
\end{abstract}

Key words: mitochondrial cardiomyopathy, chemotherapy, mutation, p53

(Intern Med 56: 1357-1361, 2017)

(DOI: 10.2169/internalmedicine.56.8076)

\section{Introduction}

Although various mitochondrial diseases such as mitochondrial myopathy, encephalopathy, lactic acidosis, and stroke-like episodes (MELAS) syndrome, myoclonic epilepsy with ragged-red fibers, chronic progressive external ophthalmoplegia, and Leigh's encephalopathy have been described, each of these conditions displays a variety of symptoms. Mitochondrial cardiomyopathy is often one of several symptoms in patients with mitochondrial diseases. The heart primarily relies on the energy produced through aerobic respiration and is one of the organs that is most affected by mitochondrial disease. In general, myocardial hypertrophy is observed to accompany a diminished left ventricular function, cardiac arrhythmias, left ventricular collapse, and heart failure in patients with these conditions. The severity of these conditions is thought to be directly linked to the mutation load, and numerous cases involving the onset of heartrelated symptoms in childhood in conjunction with mitochondrial 3,243 $\mathrm{A} \rightarrow \mathrm{G}$ mutations have been reported. In ad- dition, cardiotoxic drugs, such as anticancer drugs, can damage the myocardial mitochondria by altering matrix metalloproteases by enhancing the production of reactive oxygen species (ROS), which induces apoptosis.

In the present case, although mutations of the mitochondrial 3,243 A $\rightarrow \mathrm{G}$ gene were detected by genetic testing, the patient presented no symptoms for approximately 60 years. The turning point was the development of heart failure with anticancer drug therapy. This led to a definitive diagnosis of mitochondrial cardiomyopathy. Late onset cases of mitochondrial cardiomyopathy have not previously been reported. Furthermore, next-generation sequencing revealed several other mitochondrial DNA mutations. We report this case and discuss future challenges.

\section{Case Report}

The patient was a 62-year-old woman with a history of bilateral hearing impairment. The symptoms had appeared 5 years previously-the cause was unknown. The patient had no family history of sudden death or hearing impairment; how- 
Table 1. The Progression of the Echocardiographic Data and the Examination Findings.

\begin{tabular}{|c|c|c|c|}
\hline & Pre admission & On admission & Post admission after 3 months \\
\hline IVSTd (mm) & 12 & 10 & 10 \\
\hline PWTd (mm) & 12 & 11 & 11 \\
\hline LVDd (mm) & 39 & 48 & 41 \\
\hline LVDs (mm) & 30 & 42 & 27 \\
\hline $\mathrm{EF}(\%)$ & 47 & 27 & 46 \\
\hline $\mathrm{FS}(\%)$ & 23 & 13 & 34 \\
\hline TRPG (mm Hg) & 36 & 36 & 21 \\
\hline E/A (TMF pattern) & $\begin{array}{r}0.78 \\
\text { (abnormal relaxation) }\end{array}$ & $\begin{array}{r}1.38 \\
\text { (pseudo normalization) }\end{array}$ & $\begin{array}{r}0.80 \\
\text { (abnormal relaxation) }\end{array}$ \\
\hline $\mathrm{BNP}(\mathrm{pg} / \mathrm{mL})$ & none & 2,253 & 15 \\
\hline $\begin{array}{l}\text { Class of heart failure according to } \\
\text { NYHA classification }\end{array}$ & II & III-IV & II \\
\hline Chest X-ray & & & a \\
\hline
\end{tabular}

IVSTd: Interventricular septum thickness diameter, PWTd: Posterior left ventricular wall thickness diameter, LVDd: Left ventricular internal dimension in diastole, LVDs: Left ventricular internal dimension in systole, EF: Ejection function, FS: Fractional shortening, TRPG: Tricuspid regurgitation pressure gradient, E/A: Early diastolic filling velocity/Atrial filling velocity, TMF: Transmitral flow, BNP: brain natriuretic peptide, NYHA class: New York Heart Association classification

ever, she had a history of tobacco use for approximately 40 years. Electrocardiogram abnormalities were observed during the preoperative insertion of an applicator, which was performed as a part of a treatment for cervical cancer (stage IV). Diffuse left ventricular hypertrophy and a mildly diminished left ventricular systolic function (ejection fraction [EF]: 47\%) were observed on cardiac ultrasonography.

The patient was determined to be able to safely tolerate surgery, and first underwent surgery for cervical cancer as per the treatment guidelines. The patient underwent postoperative cisplatin chemotherapy after cervical cancer surgery. Following the completion of 5 courses of chemotherapy, dyspnea at rest and edema in both lower extremities were observed. A chest X-ray film revealed marked pulmonary congestion and cardiac ultrasonography indicated a significant decline in the left ventricular systolic function (EF: $22 \%$ ). In addition, the patient's brain natriuretic peptide level was markedly elevated at 2,253 pg/mL, and her condition was diagnosed as an acute exacerbation of chronic heart failure. Consequently, she was admitted to the Department of Cardiovascular Medicine for treatment. Diuretics and dobutamine were administered to treat the patient's heart failure. The excessive extracellular fluid that was present during chemotherapy was considered to have triggered the acute exacerbation of her heart condition.

The patient's heart condition improved on the 14th day after admission because of the drug therapy. Thereafter, she was started on beta-blockers, angiotensin-converting enzyme inhibitors, and pimobendan. The patient was discharged on the 40th day after admission with no recurrence of heart failure after cardiac ultrasonography on the 36th day of hospitalization, indicating the improvement of the left ventricular systolic function (EF: 46\%). The echocardiogram findings showed the improvement of the left ventricular function, while the examination findings showed the improvement in her class of heart failure (according to the New York Heart Association (NYHA) classification, her brain natriuretic peptide (BNP) level, and the chest X-ray findings; Table 1). Furthermore, there were no findings of increased eosinophilia during the clinical course.

Although cardiac magnetic resonance imaging (MRI), drug stress myocardial scintigraphy, and coronary angiography were conducted as part of a close examination to determine the cause of the patient's diminished left ventricular systolic function, no clear cause was identified. Cardiac MRI showed diffuse hypertrophy in the left ventricular wall (Fig. 1A, B). Late gadolinium enhancement was seen in the basal- to mid-inferolateral wall of the left ventricular subepicardial myocardium (Fig. 1C). Diffuse cardiac hypertrophy was also observed via cardiac ultrasonography, and $\alpha$-galactosidase activity levels were measured to test for cardiac Fabry disease. Although the patient's $\alpha$-galactosidase activity levels were within the normal range, as the patient was female, we were unable to eliminate the possibility of hetero-phenotypic Fabry disease. A myocardial tissue biopsy was taken from the inferior posterior wall of the left ventricle in order to reach a definitive diagnosis. Light microscopy of hematoxylin and eosin-stained sections revealed a 

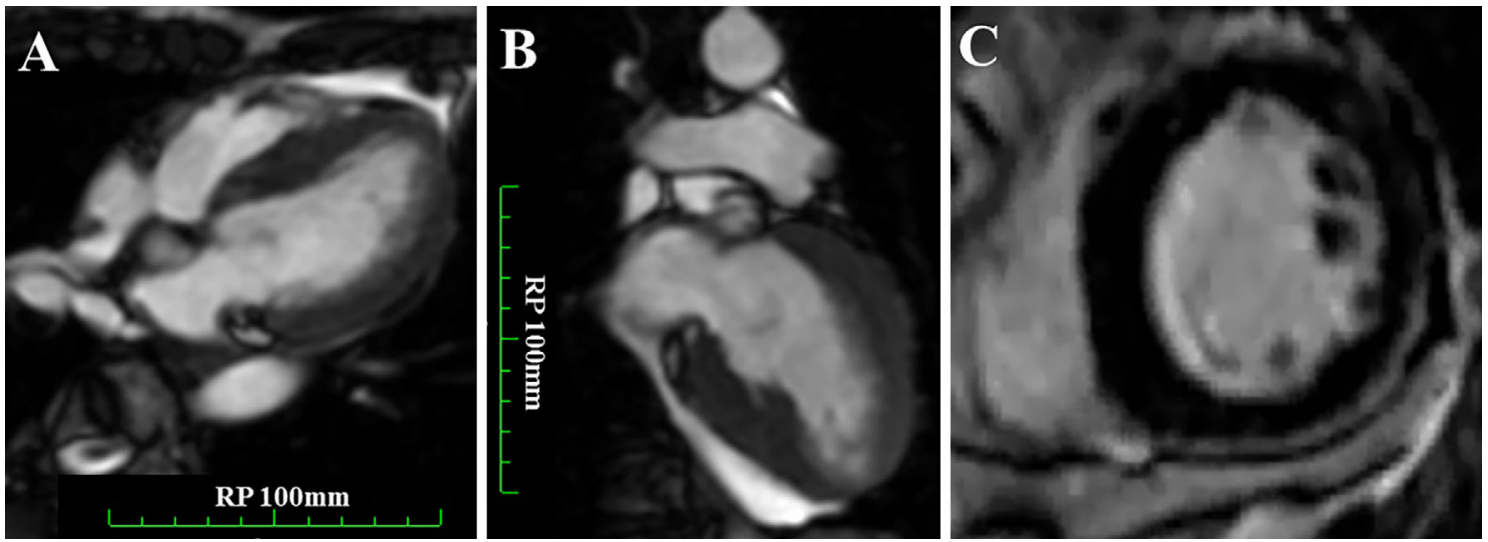

Figure 1. Cardiac magnetic resonance imaging (MRI). A and B: Perfusion images. C: Delayed gadolinium enhancement.

\section{A: Hematoxylin and eosin stain, $\times 100$}

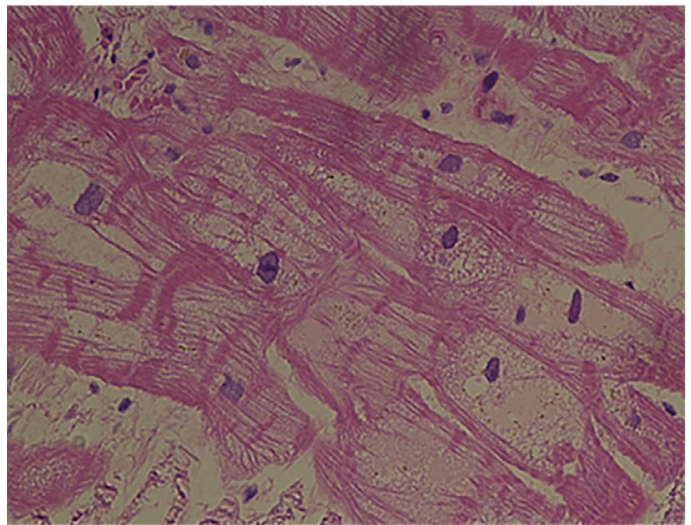

C: Periodic acid-Schiff stain, $\times 100$

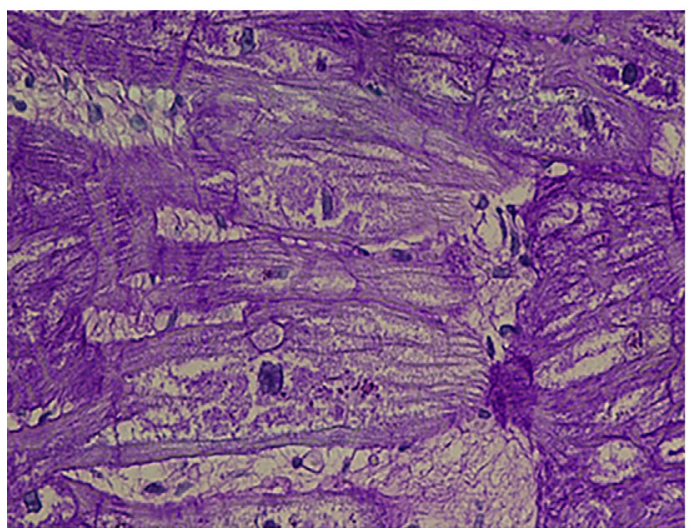

\section{B: Elastica van Gieson stain, $\times 100$}

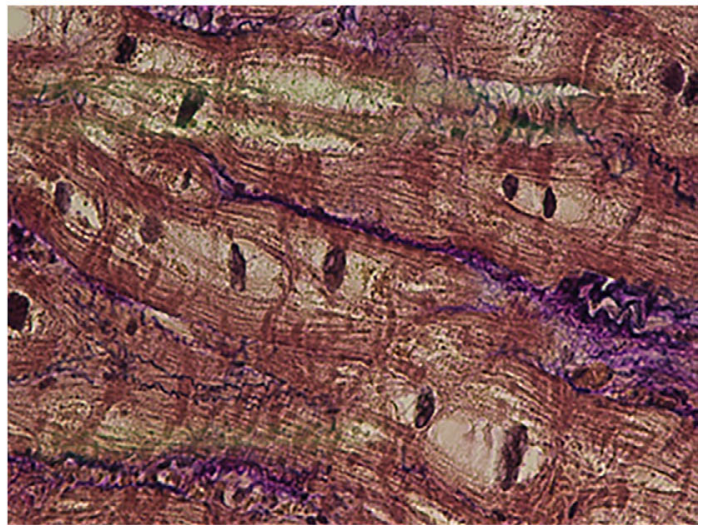

\section{D: Congo red stain, $\times 100$}

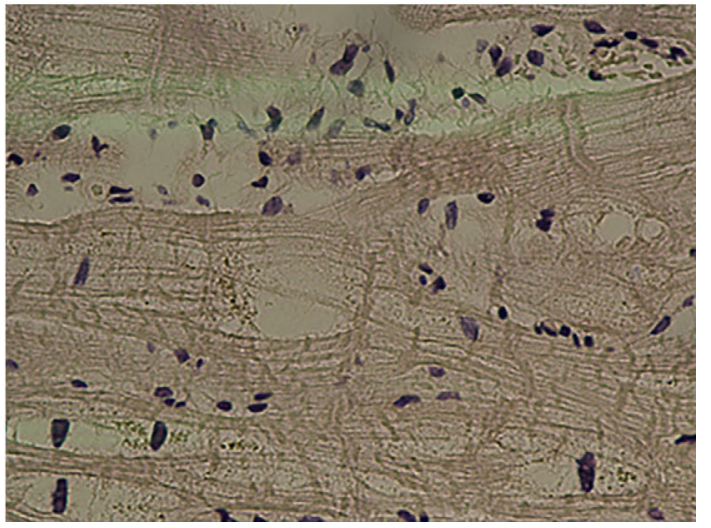

Figure 2. Light micrographs of a left ventricular endomyocardial biopsy specimen. A: Hematoxylin and Eosin staining $(\times 100)$. B: Elastica van Gieson staining $(\times 100)$. C: Periodic acid-Schiff staining $(\times 100)$. D: Congo red staining $(\times 100)$.

lack of myofibrillar disarray, clear cellular reticula, and ceramide deposition within the cellular reticula (Fig. 2A). Although other staining methods were applied, there were no significant findings (Fig. 2B, C, D). In addition, electron microscopy revealed the enargement of the mitochondrial cells (1.0-2.5 $\mu \mathrm{m}$ in diameter), which exhibited abnormal hyperplasia, central core lysis in the cristae, and abnormal concentric circular lamination (Fig. 3). Cerebral white matter lesions and global brain atrophy were observed on cranial
MRI, along with bilateral sensorineural hearing impairment. Based on these findings as well as the left ventricular biopsy findings, the patient was definitively diagnosed with MELAS syndrome. Subsequent genetic testing revealed mutations in the mitochondrial $3,243 \mathrm{~A} \rightarrow \mathrm{G}$ and $8,348 \mathrm{~A} \rightarrow \mathrm{G}$ genes. Next-generation sequencing indicated 955 individual mutations (Table 2). According to the mitochondrial abnormality observed in the left ventricular biopsy specimen and the results of the subsequent genetic testing, we hypothesize 

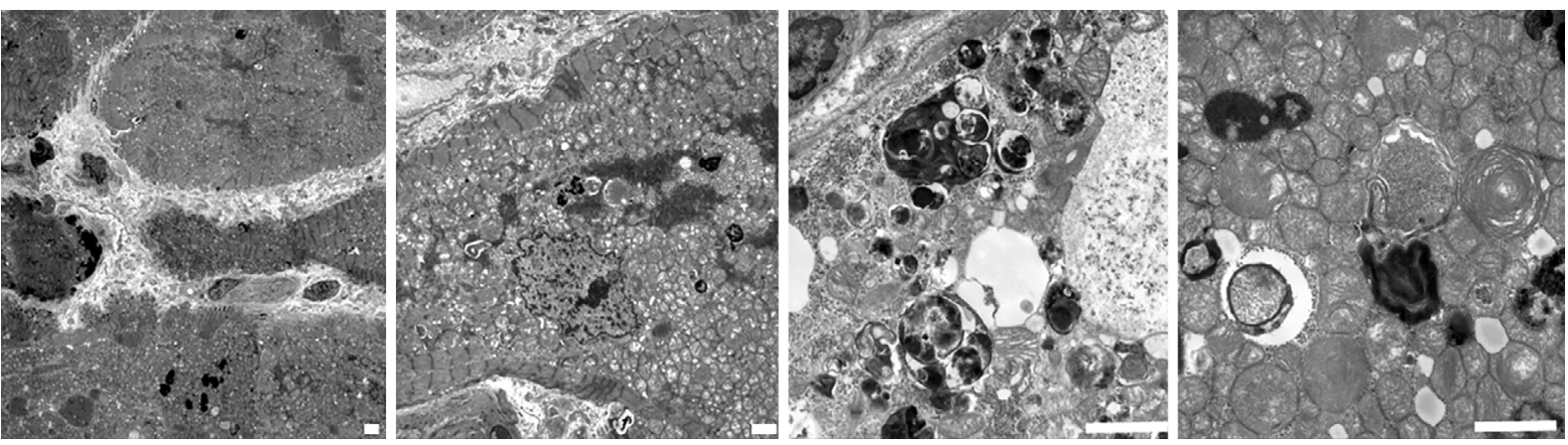

Figure 3. Electron micrographs of a left ventricular endomyocardial biopsy specimen. Electron microscopy revealed enlarged mitochondrial cells of 1.0-2.5 $\mu \mathrm{m}$ in diameter, which exhibited abnormal hyperplasia, central core lysis in the cristae, and abnormal concentric circular lamination. Scale bars: $2 \mu \mathrm{m}$.

Table 2. Subsequent Genetic Testing and Next-generation Sequencing.

\begin{tabular}{cccccccccc}
\hline No. & Chromosome & Start & End & Reference & Alternative & Zygosity & Mutation & Disease & Literature \\
\hline 1 & Mitochondria & 1,643 & 1,643 & $\mathrm{~A}$ & $\mathrm{G}$ & Heteroplasmy & $1,643 \mathrm{~A} \rightarrow \mathrm{G}$ & Late infantile onset & $(17)$ \\
2 & Mitochondria & 3,243 & 3,243 & $\mathrm{~A}$ & $\mathrm{G}$ & Heteroplasmy & $3,243 \mathrm{~A} \rightarrow \mathrm{G}$ & MELAS & $(18)$ \\
3 & Mitochondria & 4,833 & 4,833 & $\mathrm{~A}$ & $\mathrm{G}$ & Heteroplasmy & $4,833 \mathrm{~A} \rightarrow \mathrm{G}$ & Diabetes mellitus & $(19)$ \\
4 & Mitochondria & 8,348 & 8,348 & $\mathrm{~A}$ & $\mathrm{G}$ & Heteroplasmy & $8,348 \mathrm{~A} \rightarrow \mathrm{G}$ & Cardiomyopathy & $(20)$ \\
5 & Mitochondria & 9,185 & 9,185 & $\mathrm{~T}$ & $\mathrm{C}$ & Heteroplasmy & $9,285 \mathrm{~T} \rightarrow \mathrm{C}$ & Leigh's disease & $(21)$ \\
6 & Mitochondria & 11,084 & 11,084 & $\mathrm{~A}$ & $\mathrm{G}$ & Heteroplasmy & $11,084 \mathrm{~A} \rightarrow \mathrm{G}$ & MELAS & $(22)$ \\
7 & Mitochondria & 12,770 & 12,770 & $\mathrm{~A}$ & $\mathrm{G}$ & Heteroplasmy & $12,770 \mathrm{~A} \rightarrow \mathrm{G}$ & MELAS & $(23)$ \\
8 & Mitochondria & 14,693 & 14,693 & $\mathrm{~A}$ & $\mathrm{G}$ & Heteroplasmy & $14,693 \mathrm{~A} \rightarrow \mathrm{G}$ & MELAS & $(24)$
\end{tabular}

The major mitochondrial mutations among 955 individual mutations indicated by subsequent genetic testing.

that the patient's pathological condition developed due to compensated mitochondrial cardiomyopathy, which was exacerbated by decompensated heart failure with the administration of cisplatin and chemotherapy.

\section{Discussion}

MELAS syndrome is caused by the most common mitochondrial 3,243 $\mathrm{A} \rightarrow \mathrm{G}$ mutation; it is transmitted maternally. The mitochondria are the main producers of adenosine triphosphate (ATP), which supplies the energy for various cellular processes (1). Thus, MELAS syndrome patients present a various conditions of the heart and the brain with a high amount of energy. As a cardiac condition, MELAS syndrome presents ventricular hypertrophy, systolic and diastolic dysfunction, and conduction disturbance $(2,3)$. The cardiac involvement is progressive and an independent predictor of mortality in patients with mitochondrial disease (4). The mitochondrial function is a key to maintaining the heart function. The homeostasis of the mitochondria is regulated by their fusion, fission, and autophagy, which contribute to the maintenance of the energy production capacity $(5,6)$. Fission is the separation of the damaged mitochondria and is important during autophagy $(7,8)$. In addition, autophagy plays a critical role in maintaining myocardial homeostasis and the response to stressors (9). However, recent studies have revealed that these functions diminish with age (6-8). The failure of the mitochondrial function can result from the restriction of these fission and autophagy processes in the damaged mitochondria of aging cardiac tissue (10). Abnormal mitochondrial proliferation and the suppression of mitochondrial decomposition can be confirmed by electron microscopy (10).

Cisplatin produces an antitumor effect by inducing p53dependent apoptosis. Although cisplatin is a first-line drug that is used in the treatment of various cancers, such as uterine, testicular, and prostate cancer, its use is limited because of its side effects, which include the disruption of transcription, the induction of cell cycle arrest, and the induction of ROS production leading to apoptosis $(11,12)$. ROS production is particularly important with respect to apoptotic pathways. Matrix metalloproteases are altered by the enhanced ROS production, which damages the respiratory chain and induces apoptosis. Cisplatin rapidly accumulates in the mitochondria, resulting in oxidative stress and damaging mitochondrial DNA. As such, the accumulation of cisplatin is thought to result in ROS production in the mitochondria. Previous studies have reported that the accumulation of cisplatin in the mitochondria and the subsequent enhacement of ROS production resulted in damage to the mitochondria as well as toxicity to other cells (13-15). In the present case, cisplatin might have caused the increase in ROS production, which might have increased the mitochondrial damage. Although under normal conditions, the damaged mitochondria 
are broken down via fission and autophagy, abnormal mitochondrial proliferation was observed by electron microscopy. The existing decline in mitochondrial function may become further pronounced in cases involving mutations in the mitochondrial 3,243 $\mathrm{A} \rightarrow \mathrm{G}$ and 8,348 $\mathrm{A} \rightarrow \mathrm{G}$ genes. As such, in cases where the mitochondrial function is diminished, the use of drugs that are reported to exhibit cardiotoxic effects and aging can trigger the onset of mitochondrial cardiomyopathy. In the present case, regardless of the mutations that were observed in the mitochondrial 3,243 $\mathrm{A} \rightarrow \mathrm{G}$ and $8,348 \mathrm{~A} \rightarrow \mathrm{G}$ genes in the cardiomyocytes, the patient displayed no symptoms for 60 years. Numerous cases involving mitochondrial 3,243 $\mathrm{A} \rightarrow \mathrm{G}$ gene mutations and the onset of heart conditions in childhood have been reported (16); in the present case, a putative myocardial protection factor may have been responsible. Nine hundred fifty-five individual mutations were identified by next-generation sequencing. These should be further evaluated in future studies.

In cases involving mitochondrial dysfunction, even drugs with low cardiotoxicity such as cisplatin can lead to the onset of mitochondrial damage-related heart failure. Considering the increase in the number of cancer survivors, the underlying mitochondrial dysfunction caused by oxidative stress or genetic mitochondrial mutations should be considered as a cause of cardiovascular complications in patients undergoing cancer treatment.

The authors state that they have no Conflict of Interest (COI).

\section{References}

1. Limongelli G, Masarone D, D’Alessandro R, Elliott PM. Mitochondrial diseases and the heart: an overview of molecular basis, diagnosis, treatment and clinical course. Future Cardiol 8: 71-88, 2012.

2. Di Donato S. Multisystem manifestations of mitochondrial disorders. J Neurol 56: 693-710, 2009.

3. Scaglia F, Towbin JA, Craigen WJ, et al. Clinical spectrum, morbidity, and mortality in 113 pediatric patients with mitochondrial disease. Pediatrics 114: 925-931, 2004.

4. Holmgren D, Wahlander H, Eriksson BO, Oldfors A, Holme E, Tulinius M. Cardiomyopathy in children with mitochondrial disease: clinical course and cardiological findings. Eur Heart J 24: 280-288, 2003

5. Youle RJ, van der Bliek AM. Mitochondrial fission, fusion, and stress. Science 337: 1062-1065, 2012.

6. Seo AY, Joseph AM, Dutta D, Hwang JCY, Aris JP, Leeuwenburgh C. New insights into the role of mitochondria in aging: mitochondrial dynamics and more. J Cell Sci 123: 2533-2542, 2010.

7. Rubinsztein DC, Marino G, Kroemer G. Autophagy and aging. Cell 146: 682-695, 2011

8. Wang K, Klionsky DJ. Mitochondria removal by autophagy. Autophagy 7: 297-300, 2011.

9. Ikeda Y, Sciarretta S, Nagarajan N, et al. New insights into the role of mitochondrial dynamics and autophagy during oxidative stress and aging in the heart. Oxid Med Cell Longev 2014 (Epub ahead of print).

10. Hoshino A, Mita Y, Okawa Y, et al. Cytosolic p53 inhibits Parkinmediated mitophagy and promotes mitochondrial dysfunction in the mouse heart. Nat Commun 4: 2308, 2013.

11. $\mathrm{Xu} \mathrm{EY}$, Perlina $\mathrm{A}, \mathrm{Vu} \mathrm{H}$, et al. Integrated pathway analysis of rat urine metabolic profiles and kidney transcriptomic profiles to elucidate the systems toxicology of model nephrotoxicants. Chem Res Toxicol 21: 1548-1561, 2008.

12. Alborziia $\mathrm{H}$, Can $\mathrm{S}$, Holenya $\mathrm{P}$, et al. Real-time monitoring of cisplatin-induced cell death. PLoS One 6: e19714, 2011.

13. Mukhopadhyay $P$, Horvath B, Zsengeller Z, et al. Mitochondrialtargeted antioxidants represent a promising approach for prevention of cisplatin-induced nephropathy. Free Radic Biol Med 52: 497-506, 2012.

14. So H, Kim H, Lee JH, et al. Cisplatin cytotoxicity of auditory cells requires secretions of proinflammatory cytokines via activation of ERK and NF-kappaB. J Assoc Res Otolaryngol 8: 338355, 2007.

15. Hamers FP, Brakkee JH, Cavalletti E, et al. Reduced glutathione protects against cisplatin-induced neurotoxicity in rats. Cancer Res 53: 544-549, 1993.

16. Terasaki F, Tanaka M, Kawamura K, et al. A case of cardiomyopathy showing progression from the hypertrophic to the dilated form. Jpn Circ J 65: 691-694, 2001.

17. Del Mar O'Callaghan M, Emperador S, López-Gallardo E, et al. New mitochondrial DNA mutations in tRNA associated with three severe encephalopamyopathic phenotypes: neonatal, infantile, and childhood onset. Neurogenetics 13: 245-250, 2012.

18. Rajasimha HK, Chinnery PF, Samuels DC. Selection against pathogenic mtDNA mutations in a stem cell population leads to the loss of the $3243 \mathrm{~A} \rightarrow \mathrm{G}$ mutation in blood. Am J Hum Genet 82: 333-343, 2008.

19. Ohkubo E, Aida K, Chen J, et al. A patient with type 2 diabetes mellitus associated with mutations in calcium sensing receptor gene and mitochondrial DNA. Biochem Biophys Res Commun 278: $808-813$.

20. Terasaki F, Tanaka M, Kawamura K, et al. A case of cardiomyopathy showing progression from the hypertrophic to the dilated form: association of Mt8348A-->G mutation in the mitochondrial tRNA (Lys) gene with severe ultrastructural alterations of mitochondria in cardiomyocytes. Jpn Circ J 65: 691-694, 2001.

21. Bannwarth S, Procaccio V, Lebre AS, et al. Prevalence of rare mitochondrial DNAmutations in mitochondrial disorders. J Med Genet 50: 704-714, 2013.

22. Levin L, Zhidkov I, Gurman Y, et al. Functional recurrent mutations in the human mitochondrial phylogeny: dual roles in evolution and disease. Genome Biol Evol 5: 876-890, 2013.

23. Liolitsa D, Rahman S, Benton $S$, et al. Is the mitochondrial complex I ND5 gene a hot-spot for MELAS causing mutations?. Ann Neurol 53: 128-132, 2003.

24. Tzen CY, Thajeb $\mathrm{P}, \mathrm{Wu} \mathrm{TY}$, et al. MELAS with point mutations involving tRNALeu (A3243 G) and tRNAGlu (A14693 g). Muscle Nerve 28: 575-581, 2003.

The Internal Medicine is an Open Access article distributed under the Creative Commons Attribution-NonCommercial-NoDerivatives 4.0 International License. To view the details of this license, please visit (https://creativecommons.org/licenses/ by-nc-nd/4.0/).

(C) 2017 The Japanese Society of Internal Medicine

http://www.naika.or.jp/imonline/index.html 